\title{
Parametric excitations of linear systems having many degrees of freedom
}

\author{
A. H. Nayfeh and D. T. Mook \\ Department of Engineering Science and Mechanics, Virginia Polytechnic Institute and State University, \\ Blacksburg, Virginia 24061 \\ (Received 10 January 1977) \\ The method of multiple scales is used to analyze parametrically excited, linear systems having many \\ degrees of freedom and distinct natural frequencies. Explicit second-order expressions are obtained for the \\ characteristic exponents which yield the transition curves. Various combination resonances are treated. The \\ results are applied to the buckling of free-clamped columns under the influence of sinusoidally varying \\ axial loads.
}

PACS numbers: $43.40 . \mathrm{At}, 43.40 . \mathrm{Cw}$

\section{INTRODUCTION}

The present paper is concerned with systems governed by equations having the form

$$
\ddot{u}_{n}+\omega_{n}^{2} u_{n}+2 \epsilon(\cos \omega t) \sum_{n=1}^{N} f_{n m} u_{m}=0 \text {, }
$$

where $n=1,2, \cdots, N$; $N$ may be any positive integer; $\epsilon$ is a small parameter; dots indicate differentiation with respect to time; and the natural frequencies $\omega_{n}$ are distinct and ordered so that $\omega_{n}<\omega_{n+1}$. Similar systems were considered in a number of earlier studies; the following brief discussion of representative examples provides a background for the present paper.

$\mathrm{Hsu}^{1,2}$ obtained approximate solutions for various resonant situations and included the case when the natural frequencies are not distinct. Yamamoto and Saito ${ }^{3}$ obtained approximate solutions for various resonant situations. Moreover, they compared their analytical results with their results obtained experimentally and by using an analog computer. Mettler ${ }^{4}$ considered a system in which the periodic coefficients multiply polynomials in the unknown functions. The analyses in these three studies were based on the method of averaging. (Nayfeh gives a description of the various perturbation methods.) Only two terms in the approximation, which includes expressions for the transition curves in the $\epsilon-\omega$ plane between stable and unstable solutions, were obtained.

Using analog simulation, Sugiyama et al. ${ }^{6}$ found combination resonances in the responses of clamped-f ree columns to periodic tangential loads. Iwatsubo et al. ${ }^{7}$ demonstrated experimentally the existence of combination resonances in the responses of clamped-clamped and clamped-simply supported columns. Iwatsubo et al. ${ }^{8}$ used Galerkin's method in conjunction with Hsu's stability criteria to study various resonances in the responses of columns to periodic axial loads.

Fu and Nemat-Nasser ${ }^{9}$ used Hill's method to obtain the first two terms in the expressions for the transition curves for various resonant conditions. They included the case in which not all the natural frequencies are distinct.

Keeping with the idea of averaging, one could use, for example, the method of Struble, the method of Krylov, Bogoliubov, and Mitropolski, or Lie series and trans- forms to obtain higher-order terms in the approximations. However, to the authors' knowledge, none of these methods have been used.

Valeev ${ }^{10-12}$ obtained three-term approximations of the transition curves for various similar systems. He used the Laplace transformation to convert the set of differential equations into a set of linear difference equations and solved the latter using Hill's method.

The method of multiple scales is an alternative to the methods mentioned above, though it has not been applied to this problem before. In the present paper, this method is used to construct three-term approximations of the solution which yield explicit expressions for the characteristic exponents and the transition curves. Several of the resonant situations considered in the present paper were also considered by Hsu and Valeev, and the present results are shown to agree with theirs. In addition, a number of other resonant situations are considered in the present paper for the first time.

\section{STRAIGHTFORWARD EXPANSION}

The different possible resonant conditions can be exhibited by a straightforward expansion having the form

$$
u_{n}(t ; \epsilon) \sim u_{n} 0(t)+\epsilon u_{n}(t)+\cdots
$$

Substituting Eq. (2) into Eq. (1) and equating coefficients of like powers of $\epsilon$ yields

$$
\begin{aligned}
& \ddot{u}_{n^{0}}+\omega_{n}^{2} u_{n^{0}}=0, \\
& \ddot{u}_{n^{1}}+\omega_{n}^{2} u_{n^{1}}=-\sum_{r} f_{n r} u_{r 0}[\exp (i \omega t)+\mathrm{cc}], \\
& \ddot{u}_{n^{2}}+\omega_{n}^{2} u_{n^{2}}=-\sum_{r} f_{n r} u_{r 1}[\exp (i \omega t)+c c],
\end{aligned}
$$

where cc represents the complex conjugate of the preceding terms. form

The general solution of Eq. (3) can be written in the

$$
u_{n} 0=A_{n} \exp \left(i \omega_{n} t\right)+\mathrm{cc},
$$

where $A_{n}$ is a complex constant.

Substituting Eq. (6) into Eq. (4) yields 
$\ddot{u}_{n}+\omega_{n}^{2} u_{n}=-\sum_{r} f_{n r} A_{r}\left\{\exp \left[i\left(\omega_{r}+\omega\right) t\right]+\exp \left[i\left(\omega_{r}-\omega\right) t\right]\right\}+c c$.

A particular solution of Eq. (7) can be written in the form

$u_{n^{1}}=\sum_{r} f_{n r} A_{r}\left[\frac{\exp \left[i\left(\omega_{r}+\omega\right) t\right]}{\left(\omega_{r}+\omega\right)^{2}-\omega_{n}^{2}}+\frac{\exp \left[i\left(\omega_{r}-\omega\right) t\right]}{\left(\omega_{r}-\omega\right)^{2}-\omega_{n}^{2}}\right]+\mathbf{c c}$.

(8)
Substituting Eq. (8) into Eq. (5) yields

$$
\begin{aligned}
\ddot{u}_{n^{2}}+\omega_{n}^{2} u_{n^{2}}= & -\sum_{r} \sum_{s} f_{n r} f_{r s} A_{s}\left[\frac{\exp \left[i\left(\omega_{s}+2 \omega\right) t\right]+\exp \left(i \omega_{s} t\right)}{\left(\omega_{s}+\omega\right)^{2}-\omega_{r}^{2}}\right. \\
& \left.+\frac{\exp \left[i\left(\omega_{s}-2 \omega\right) t\right]+\exp \left(i \omega_{s} t\right)}{\left(\omega_{s}-\omega\right)^{2}-\omega_{r}^{2}}\right]+\mathrm{cc}
\end{aligned}
$$

A particular solution of Eq. (9) can be written in the form

$$
\begin{aligned}
u_{n^{2}}= & \sum_{r} \sum_{s} f_{n r} f_{r s} A_{s}\left\{\frac{\exp \left[i\left(\omega_{s}+2 \omega\right) t\right]}{\left.\left[\left(\omega_{s}+2 \omega\right)^{2}-\omega_{n}^{2}\right]\left[\omega_{s}+\omega\right)^{2}-\omega_{r}^{2}\right]}+\frac{\exp \left[i\left(\omega_{s}-2 \omega\right) t\right]}{\left.\left[\left(\omega_{s}-2 \omega\right)^{2}-\omega_{n}^{2}\right]\left[\omega_{s}-\omega\right)^{2}-\omega_{r}^{2}\right]}\right\} \\
& +\sum_{r} \sum_{\substack{s \\
s \neq n}} f_{n r} f_{r s} A_{s}\left[\frac{1}{\left(\omega+\omega_{s}\right)^{2}-\omega_{r}^{2}}+\frac{1}{\left(\omega-\omega_{s}\right)^{2}-\omega_{r}^{2}}\right] \frac{\exp \left(i \omega_{s} t\right)}{\omega_{s}^{2}-\omega_{n}^{2}} \\
& +\frac{1}{2} i \sum_{r} f_{n r} f_{r n} A_{n}\left[\frac{1}{\left(\omega+\omega_{n}\right)^{2}-\omega_{r}^{2}}+\frac{1}{\left(\omega-\omega_{n}^{2}\right)-\omega_{r}^{2}}\right] \frac{t \exp \left(i \omega_{n} t\right)}{\omega_{n}}+\mathbf{c c .}
\end{aligned}
$$

It follows from Eqs. (6) and (10) that expansion (2) is only valid for short times because $\lim _{t \rightarrow \infty}\left(u_{n^{2}} / u_{n^{0}}\right)=\infty$; this is the result of the secular terms in $u_{n} 2$ which contain the factors $t \exp \left( \pm i \omega_{n} t\right)$. It follows from Eqs. (8) and (10) that expansion (2) is not valid if $p \omega \approx \omega_{n} \pm \omega_{m}$, where $p, n$, and $m$ are integers, because when such resonant combinations of frequencies exist some terms in $u_{n^{1}}, u_{n^{2}}$, etc., may contain small divisors. As mentioned in the Introduction, the existence of such combination resonances has been demonstrated both experimentally and by analog simulation. ${ }^{3,6,7}$

In order to eliminate the troublesome secular and small-divisor terms, one must modify the straightforward procedure. In this paper, the modification is accomplished by using the method of multiple scales.

\section{FIRST-ORDER EXPANSIONS}

Following the method of multiple scales, one seeks a uniformly valid expansion having the form

$$
u_{n}(t ; \epsilon) \sim u_{n 0}\left(T_{0}, T_{1}, T_{2}\right)+\epsilon u_{n^{1}}\left(T_{0}, T_{1}, T_{2}\right)+\cdots++,
$$

where $T_{0}=t$ is the so-called fast scale which is associated with changes occurring at the frequencies $\omega$ and $\omega_{n}$, while $T_{1}=\epsilon t$ and $T_{2}=\epsilon^{2} t$ are the so-called slow scales which are associated with modulations in amplitudes and phases resulting from the parametric excitation and occurring at frequencies much lower than $\omega$ and $\omega_{n}$.

Substituting expansion (11) into Eq. (1) and equating coefficients of like powers of $\epsilon$ yields

$$
\begin{aligned}
& D_{0}^{2} u_{n 0}+\omega_{n}^{2} u_{n}=0 \\
& D_{0}^{2} u_{n} 1+\omega_{n}^{2} u_{n}=-2 D_{0} D_{1} u_{n 0}-\sum_{r} f_{n r} u_{r 0}\left[\exp \left(i \omega T_{0}\right)+\mathrm{cc}\right],
\end{aligned}
$$

and

$$
\begin{aligned}
D_{0}^{2} u_{n^{2}}+\omega_{n}^{2} u_{n^{2}}= & -2 D_{0} D_{2} u_{n} 0-D_{1}^{2} u_{n 0}-2 D_{0} D_{1} u_{n^{1}} \\
& -\sum_{r} f_{n r} u_{r 1}\left[\exp \left(i \omega T_{0}\right)+\mathrm{cc}\right]
\end{aligned}
$$

where $D_{m}=\partial / \partial T_{m}$.

The general solution of Eq. (12) can be written in the form

$$
u_{n 0}=A\left(T_{1}, T_{2}\right) \exp \left(i \omega_{n} T_{0}\right)+\mathbf{c c},
$$

where the complex functions $A_{n}$ are undetermined at this level of approximation; they are determined by eliminating the terms from Eqs. (13) and (14) which produce the troublesome secular and small-divisor terms in $u_{n^{1}}$ and $u_{n}$.

Substituting Eq. (15) into Eq. (13) yields

$$
\begin{aligned}
& D_{0}^{2} u_{n^{1}}+\omega_{n}^{2} u_{n 1}=-2 i \omega_{n} D_{1} A_{n} \exp \left(i \omega_{n} T_{0}\right) \\
& \quad-\sum_{r} f_{n r} A_{r}\left\{\exp \left[i\left(\omega_{r}+\omega\right) T_{0}\right]+\exp \left[i\left(\omega_{r}-\omega\right) T_{0}\right]\right\}+c c .
\end{aligned}
$$

Now $A_{n}$ is to be chosen in such a way as to eliminate troublesome terms from $u_{n 1}$. This choice depends on the resonant combinations of frequencies; in this section, five different cases are considered.

\section{A. The case $\omega$ away from $\omega_{q} \pm \omega_{p}$}

In this case, $\omega$ is away from $\omega_{q} \pm \omega_{p}$ for all possible values of $p$ and $q$. Hence, small divisors cannot appear, and the troublesome term will be eliminated from $u_{n^{1}}$ if for all $n$

$$
D_{1} A_{n}=0 \text { or } A_{n}=A_{n}\left(T_{2}\right) \text {. }
$$

Consequently, a particular solution of Eq. (16) can be written in the form

$u_{n 1}=\sum_{r} f_{n r} A_{r}\left\{\frac{\exp \left[i\left(\omega_{r}+\omega\right) T_{0}\right]}{\left(\omega_{r}+\omega\right)^{2}-\omega_{n}^{2}}+\frac{\exp \left[i\left(\omega_{r}-\omega\right) T_{0}\right]}{\left(\omega_{r}-\omega\right)^{2}-\omega_{n}}\right\}+c c$.

\section{B. The case $\omega$ near $\omega_{p}+\omega_{q}$}

In this case $\omega$ is near $\omega_{p}+\omega_{q}$, and there are no other resonances to this order. To express the nearness of 
$\omega$ to $\omega_{p}+\omega_{q}$, one can introduce the detuning parameter $\sigma$ which is defined by the following equation:

$$
\omega=\omega_{p}+\omega_{q}+\epsilon \sigma
$$

Then, using Eq. (19), one can write

$$
\left(\omega-\omega_{q}\right) T_{0}=\omega_{p} T_{0}+\sigma T_{1}
$$

and

$$
\left(\omega-\omega_{p}\right) T_{0}=\omega_{q} T_{0}+\sigma T_{1} .
$$

Then it follows from Eq. (16) that the troublesome terms are eliminated from $u_{p 1}$ if

$$
2 i \omega_{p} D_{1} A_{p}+f_{p q} \bar{A}_{q} \exp \left(i \sigma T_{1}\right)=0,
$$

the troublesome terms are eliminated from $u_{q^{1}}$ if

$$
2 i \omega_{q} D_{1} A_{q}+f_{q p} \bar{A}_{p} \exp \left(i \sigma T_{1}\right)=0,
$$

and the troublesome terms are eliminated from $u_{n}$, where $n \neq p$ and $q$, if Eq. (17) is satisfied.

Equations (22) and (23) admit nontrivial solutions having the form

$$
A_{p}=a_{p} \exp \left(-i \lambda T_{1}\right) \text { and } A_{q}=a_{q} \exp \left[i(\bar{\lambda}+\sigma) T_{1}\right],
$$

where $a_{p}$ and $a_{q}$ are complex functions of $T_{2}$,

$$
\lambda=-\frac{1}{2}\left[\sigma \pm\left(\sigma^{2}-\Lambda_{p q}\right)^{1 / 2}\right],
$$

and

$$
\Lambda_{p Q}=f_{p q} f_{q p} / \omega_{p} \omega_{a} \text {. }
$$

It follows from Eqs. (24) and (25) that $A_{p}$ and $A_{q}$ are bounded if, and only if,

$$
\sigma^{2} \geq \Lambda_{p q} \text {. }
$$

When $\Lambda_{p q}>0$ (i.e., when $f_{p q}$ and $f_{q p}$ have the same sign), the transition curves in the $\epsilon-\omega$ plane separating stable from unstable solutions are defined by the following:

$$
\omega=\omega_{p}+\omega_{q} \pm \epsilon\left(f_{p q} f_{q p} / \omega_{p} \omega_{q}\right)^{1 / 2}+O\left(\epsilon^{2}\right)
$$

which agrees with the result of Hsu. ${ }^{1}$ When $p=q$, Eq.

(28) reduces to

$$
\omega=2 \omega_{p} \pm \epsilon\left(f_{p p} / \omega_{p}\right)+O\left(\epsilon^{2}\right)
$$

which is the known result for the Mathieu equation.

In this case $u_{p 1}, u_{q 1}$, and $u_{n^{1}}$ for $n \neq p$ and $q$ are given by Eq. (18) with the troublesome terms being deleted.

\section{The case $\omega$ near $\omega_{q}-\omega_{p}$}

In this case $\omega$ is near $\omega_{q}-\omega_{p}$ and there are no other resonances to this order. The results can be obtained from those above by simply changing the sign of $\omega_{p}$. For this case, unstable solutions occur only when $f_{p q}$ and $f_{a p}$ have different signs.

\section{The case $\omega$ near $\omega_{p}+\omega_{q}$ and $\omega_{s}-\omega_{q}$}

In this case $\omega$ is simultaneously near $\omega_{p}+\omega_{a}$ and $\omega_{s}$ $-\omega_{q}$, and there are no other resonances to this order. To express the nearness of $\omega$ to $\omega_{p}+\omega_{q}$ and $\omega_{s}-\omega_{q}$, one can introduce the two detuning parameters which are defined in the following equations:

$$
\omega=\omega_{p}+\omega_{q}+\epsilon \sigma_{1} \text { and } \omega=\omega_{s}-\omega_{q}+\epsilon \sigma_{2} .
$$

Then it follows that the troublesome terms are eliminated if

$$
\begin{aligned}
& 2 i \omega_{p} D_{1} A_{p}+f_{p q} \bar{A}_{q} \exp \left(i \sigma_{1} T_{1}\right)=0, \\
& 2 i \omega_{q} D_{1} A_{q}+f_{q p} \bar{A}_{p} \exp \left(i \sigma_{1} T_{1}\right)+f_{q s} A_{s} \exp \left(-i \sigma_{2} T_{1}\right)=0, \\
& 2 i \omega_{s} D_{1} A_{s}+f_{s q} A_{q} \exp \left(i \sigma_{2} T_{1}\right)=0,
\end{aligned}
$$

and for $n \neq p, q$, and $s$, the $A_{n}$ satisfy Eq. (17). Equations (31) $-(33)$ admit nontrivial solutions having the form

$$
\begin{aligned}
& A_{p}=a_{p} \exp \left[-i\left(\bar{\lambda}-\sigma_{1}\right) T_{1}\right], \\
& A_{q}=a_{a} \exp \left(i \lambda T_{1}\right),
\end{aligned}
$$

and

$$
A_{s}=a_{s} \exp \left[i\left(\lambda+\sigma_{2}\right) T_{1}\right],
$$

where $a_{p}, a_{q}$, and $a_{s}$ are complex functions of $T_{2}$ and

$$
\lambda+\frac{1}{4} \Lambda_{p q}\left(\lambda+\sigma_{1}\right)^{-1}-\frac{1}{4} \Lambda_{q s}\left(\lambda+\sigma_{2}\right)^{-1}=0 .
$$

Equation (35) is a cubic equation for $\lambda$ and has closedform solutions. The transition curves correspond to the values of $\epsilon$ and $\omega$ for which $\lambda$ has two real roots.

In this case, $u_{p 1}, u_{q^{1}}, u_{s^{1}}$, and $u_{n^{1}}$ for $n \neq p, q$, and $s$ are given by Eq. (18) with the troublesome terms being deleted.

We note that, as $\epsilon$ vanishes, both resonances cannot exist simultaneously; either $\sigma_{2}$ or $\sigma_{1}$ becomes large, depending on whether $\omega$ approaches $\omega_{p}+\omega_{q}$ or $\omega_{s}-\omega_{q}$. Thus, as $\epsilon$ vanishes, one expects Eq. (35) to reduce to the results for a single resonance. To see that this occurs, we consider, for example, $\omega$ to be near $\omega_{p}+\omega_{q}$. Consequently, $\sigma_{2}$ becomes large and Eq. (35) reduces to

$$
\lambda+\frac{1}{4} \Lambda_{p q}\left(\lambda+\sigma_{1}\right)^{-1}=0 .
$$

It follows that

$$
\lambda=-\frac{1}{2}\left[\sigma_{1} \pm\left(\sigma_{1}^{2}-\Lambda_{p q}\right)^{1 / 2}\right],
$$

which agrees with Eq. (25).

\section{E. The case $\omega$ near $\omega_{q}-\omega_{p}$ and $\omega_{s}-\omega_{q}$}

In this case $\omega$ is simultaneously near $\omega_{q}-\omega_{p}$ and $\omega_{s}$ $-\omega_{q}$, and there are no other resonances to this order. The results can be obtained from those directly above by simply changing the sign of $\omega_{p}$.

\section{SECOND-ORDER EXPANSIONS}

Substituting Eqs. (15) and (18) into Eq. (14) yields

$$
\begin{aligned}
D_{0}^{2} u_{n^{2}}+\omega_{n}^{2} u_{n^{2}}=-\left(2 i \omega_{n} D_{2} A_{n}+D_{1}^{2} A_{n}\right) \exp \left(i \omega_{n} T_{0}\right) \\
-\sum_{r} \sum_{s} f_{n r} f_{r s} A_{s}\left\{\frac{\exp \left[i\left(\omega_{s}+2 \omega\right) T_{0}\right]+\exp \left(i \omega_{s} T_{0}\right)}{\left(\omega_{s}+\omega\right)^{2}-\omega_{r}^{2}}\right. \\
\left.+\frac{\exp \left[i\left(\omega_{s}-2 \omega\right) T_{0}\right]+\exp \left(i \omega_{s} T_{0}\right)}{\left(\omega_{s}-\omega\right)^{2}-\omega_{r}^{2}}\right\}+\mathrm{cc}+\mathrm{NTT},
\end{aligned}
$$

where NTT stands for terms which cannot produce troublesome terms in $u_{n^{2}}$ under any of the resonant conditions being considered. As in the previous section, different cases need to be considered. 


\section{A. The case $\omega$ away from $\omega_{q} \pm \omega_{p}$}

Here three subcases are considered: $2 \omega$ away from $\omega_{l} \pm \omega_{k}, 2 \omega$ near $\omega_{l}+\omega_{k}$, and $2 \omega$ near $\omega_{l}-\omega_{k}$. Other resonances, such as $2 \omega$ simultaneously near $\omega_{l}-\omega_{k}$ and $\omega_{m}$ $-\omega_{l}$, can be treated in the same manner as the cases being considered, but the results are not presented here.

In this case $A_{n}$ is a function of $T_{2}$ only [recall Eq. (17)].

When $2 \omega$ is away from $\omega_{l} \pm \omega_{k}$ for all possible values of $l$ and $k$, troublesome terms are eliminated if

$$
i D_{2} A_{n}+\chi_{n} A_{n}=0 \text {, }
$$

where

$$
2 \omega_{n} \chi_{n}=\sum_{r} f_{n r} f_{r n}\left[\frac{1}{\left(\omega_{n}+\omega\right)^{2}-\omega_{r}^{2}}+\frac{1}{\left(\omega_{n}-\omega\right)^{2}-\omega_{r}^{2}}\right] .
$$

The solution of Eq. (37) can be written in the form

$$
A_{n}=a_{n} \exp \left(i \chi_{n} T_{2}\right) \text {, }
$$

where $a_{n}$ is a complex constant. Consequently, to second order, every mode which is not part of either of the resonant combinations $\omega$ near $\omega_{q} \pm \omega_{p}$ or $2 \omega$ near $\omega_{l} \pm \omega_{k}$ is bounded for all times.

When $2 \omega$ is near $\omega_{l}+\omega_{k}$, it is convenient to introduce the detuning parameter defined in the following:

$$
2 \omega=\omega_{t}+\omega_{k}+\epsilon^{2} \sigma .
$$

Then the troublesome terms are eliminated if

$$
\begin{aligned}
& i D_{2} A_{k}+\chi_{k} A_{k}+\mu_{k l} \bar{A}_{l} \exp \left(i \sigma T_{2}\right)=0, \\
& i D_{2} A_{l}+\chi_{l} A_{l}+\mu_{l k} \bar{A}_{k} \exp \left(i \sigma T_{2}\right)=0,
\end{aligned}
$$

and for $n \neq l$ or $k$ the $A_{n}$ satisfy Eq. (37). In Eqs. (41) and (42),

$$
\mu_{k l}\left(\omega_{k}, \omega_{l}\right)=\frac{1}{2 \omega_{k}} \sum_{r} f_{k r} f_{r l}\left[\frac{1}{\left(\omega_{l}-\omega\right)^{2}-\omega_{k}^{2}}\right] \text {. }
$$

Equations (41) and (42) admit nontrivial solutions having the form

$$
A_{k}=a_{k} \exp \left(i \lambda T_{2}\right)
$$

and

$$
A_{l}=a_{\imath} \exp \left[-i(\bar{\lambda}-\sigma) T_{2}\right],
$$

where $a_{k}$ and $a_{l}$ are complex constants and

$$
\lambda^{2}-\lambda\left(\sigma-\chi_{l}+\chi_{k}\right)+\chi_{k}\left(\sigma-\chi_{l}\right)+\mu_{k l} \mu_{l k}=0 \text {. }
$$

Hence, the transition curves correspond to the vanishing of the discriminant of Eq. (45); they correspond to

$$
\sigma=\chi_{k}+\chi_{l} \pm 2\left(\mu_{k l} \mu_{l k}\right)^{1 / 2} \text {. }
$$

Combining Eqs. (40) and (46) leads to the following definition of the transition curves in the $\epsilon-\omega$ plane:

$$
\omega=\frac{1}{2}\left(\omega_{l}+\omega_{k}\right)+\epsilon^{2}\left[\frac{1}{2}\left(\chi_{k}+\chi_{l}\right) \pm\left(\mu_{k l} \mu_{l k}\right)^{1 / 2}\right] .
$$

Unstable solutions occur only when $\mu_{k l}$ and $\mu_{t k}$ have opposite signs.

The case $2 \omega$ near $\omega_{l}-\omega_{k}$ can be obtained from the above results by simply changing the sign of $\omega_{k}$.

\section{B. The case $\omega$ near $\omega_{p}+\omega_{q}$}

In this case $\omega$ is near $\omega_{p}+\omega_{q}$, and there are no other resonances. Mor eover, $A_{\rho}$ and $A_{q}$ are functions of $T_{1}$ and $T_{2}$; consequently, troublesome terms are eliminated if

$$
\begin{aligned}
& 2 i \omega_{p} D_{2} A_{p}+D_{1}^{2} A_{p}+2 \omega_{p} \hat{\chi}_{p} A_{p}=0, \\
& 2 i \omega_{q} D_{2} A_{q}+D_{1}^{2} A_{q}+2 \omega_{q} \hat{\chi}_{q} A_{q}=0,
\end{aligned}
$$

and for $n \neq p$ or $q$ the $A_{n}$ satisfy Eq. (37). In Eqs. (48) and (49),

$$
\begin{aligned}
2 \omega_{p} \hat{\mathrm{X}}_{p} & =\sum_{r} f_{p r} f_{r p}\left[\left(\omega_{p}+\omega\right)^{2}-\omega_{r}^{2}\right]^{-1} \\
& +\sum_{r \neq q} f_{p r} f_{r p}\left[\left(\omega_{p}-\omega\right)^{2}-\omega_{r}^{2}\right]^{-1} .
\end{aligned}
$$

Comparing Eqs. (38) and (50), one sees $\hat{\chi}_{p}$ is formed by removing the terms containing small divisors from $\chi_{p}$.

It is convenient, for reasons which are given below, to combine Eqs. (22) and (48) as well as Eqs. (23) and (49) to form single equations in terms of the original time scale. To accomplish this, one may use Eqs. (22) and (23) to obtain

$$
D_{1}^{2} A_{p}=\frac{1}{4} \Lambda_{p q} A_{p}-\left(\sigma f_{p q} / 2 \omega_{p}\right) \bar{A}_{q} \exp \left(i \sigma T_{1}\right),
$$

and

$$
D_{1}^{2} A_{q}=\frac{1}{4} \Lambda_{p q} A_{q}-\left(\sigma f_{q p} / 2 \omega_{q}\right) \bar{A}_{p} \exp \left(i \sigma T_{1}\right) .
$$

Substituting Eqs. (51) and (52) into Eqs. (48) and (49) yields

$$
\begin{aligned}
& 2 i \omega_{p} D_{2} A_{p}+\left(\frac{1}{4} \Lambda_{p q}+2 \omega_{p} \hat{\chi}_{p}\right) A_{p} \\
& -\left(\sigma f_{p q} / 2 \omega_{p}\right) \bar{A}_{q} \exp \left(i \sigma T_{1}\right)=0,
\end{aligned}
$$

and

$$
\begin{aligned}
& 2 i \omega_{q} D_{2} A_{q}+\left(\frac{1}{4} \Lambda_{p q}+2 \omega_{q} \hat{\chi}_{q}\right) A_{q} \\
& -\left(\sigma f_{q p} / 2 \omega_{q}\right) \bar{A}_{p} \exp \left(i \sigma T_{1}\right)=0 .
\end{aligned}
$$

It can easily be shown that Eqs. (22) and (53) are the first two terms in a multiple-scales expansion of

$$
\begin{aligned}
2 i \omega_{p} \frac{d A_{p}}{d t} & +\epsilon f_{p q}\left(1-\frac{\epsilon \sigma}{2 \omega_{p}}\right) \bar{A}_{p} \exp (i \epsilon \sigma t)+\epsilon^{2}\left(\frac{1}{4} \Lambda_{p q}\right. \\
& \left.+2 \omega_{p} \hat{\chi}_{p}\right) A_{p}=0 .
\end{aligned}
$$

Similarly, Eqs. (23) and (54) are the first two terms in a multiple-scales expansion of

$$
\begin{aligned}
2 i \omega_{a} \frac{d A_{q}}{d t} & +\epsilon f_{a p}\left(1-\frac{\epsilon \sigma}{2 \omega_{q}}\right) \bar{A}_{p} \exp (i \epsilon \sigma t)+\epsilon^{2}\left(\frac{1}{4} \Lambda_{b q}\right. \\
& \left.+2 \omega_{q} \hat{\chi}_{q}\right) A_{q}=0 .
\end{aligned}
$$

Equations (55) and (56) admit a nontrivial solution having the form

$$
A_{p}=a_{p} \exp [i \epsilon(\lambda+\sigma) t] \text { and } A_{q}=a_{q} \exp (-i \epsilon \bar{\lambda} t),
$$

where $a_{p}$ and $a_{q}$ are complex constants and

$$
\lambda^{2}+\left(\sigma+\epsilon \gamma_{1}\right) \lambda+\frac{1}{4} \Lambda_{p q}+\epsilon \sigma \gamma_{2}=0 \text {. }
$$

In Eq. (58),

$$
\gamma_{1}=\frac{1}{8} \Lambda_{p q}\left(1 / \omega_{q}-1 / \omega_{p}\right)+\hat{\chi}_{q}-\hat{\chi}_{p}
$$

and 


$$
\gamma_{2}=\hat{\chi}_{Q}-\frac{1}{8}\left(\Lambda_{p q} / \omega_{p}\right)
$$

Solving Eq. (58) gives

$$
\lambda=-\frac{1}{2}\left\{\sigma+\epsilon \gamma_{1} \pm\left[\left(\sigma+\epsilon \gamma_{1}\right)^{2}-\Lambda_{p q}-4 \epsilon \sigma \gamma_{2}\right]\right\}^{1 / 2} .
$$

The transition curves correspond to the vanishing of the radical in Eq. (61); that is

$$
\sigma+\epsilon \gamma_{1}= \pm\left(\Lambda_{p q}\right)^{1 / 2}\left(1+2 \epsilon \sigma \gamma_{2} / \Lambda_{p q}\right)+O\left(\epsilon^{2}\right) \text {. }
$$

Eliminating $\sigma$ from Eqs. (19) and (62) yields

$$
\begin{aligned}
\omega= & \omega_{p}+\omega_{q} \pm \epsilon\left(\Lambda_{p q}\right)^{1 / 2} \\
& -\frac{1}{2} \epsilon^{2}\left\{\frac{1}{4} \Lambda_{p q}\left(\frac{1}{\omega_{p}}+\frac{1}{\omega_{q}}\right)-\sum_{r} \omega_{r}\left[\frac{\Lambda_{r q}}{\left(\omega_{p}+2 \omega_{q}\right)^{2}-\omega_{r}^{2}}\right.\right. \\
& \left.\left.+\frac{\Lambda_{r p}}{\left(2 \omega_{p}+\omega_{q}\right)^{2}-\omega_{r}^{2}}\right]-\sum_{r \neq p} \frac{\omega_{r} \Lambda_{r q}}{\omega_{p}^{2}-\omega_{r}}-\sum_{r \neq q} \frac{\omega_{r} \Lambda_{r p}}{\omega_{q}^{2}-\omega_{r}^{2}}\right\}+O\left(\epsilon^{3}\right) .
\end{aligned}
$$

In comparing Eq. (63) with the results of Valeev, ${ }^{10,11}$ one should note that the sign of the second term on the right side of Eq. (5.3) in Ref. 11 should be changed to a minus. Then this equation will be consistent with Eq. (2.6) given earlier by Valeev (Ref. 10) and Eq. (63) of the present paper.

When $p=q$, Eq. (63) reduces to

$\omega=2 \omega_{p} \pm \epsilon \frac{f_{p p}}{\omega_{p}}-\epsilon^{2}\left(\frac{f_{p p}^{2}}{4 \omega_{p}^{3}}-\sum_{r} \frac{\omega_{r} \Lambda_{r p}}{9 \omega_{p}^{2}-\omega_{r}^{2}}-\sum_{r \neq p} \frac{\omega_{r} \Lambda_{r p}}{\omega_{p}-\omega_{r}^{2}}\right)$.

If $f_{p r}=0$ for $r \neq p$, then Eq. (64) reduces to

$$
\omega=2 \omega_{p} \pm \epsilon \frac{f_{p p}}{\omega_{p}}-\epsilon^{2} \frac{f_{p p}^{2}}{8 \omega_{p}^{3}}+O\left(\epsilon^{3}\right)
$$

which is the known result for the Mathieu equation.

Note that one cannot expand Eq. (61) for small $\epsilon$ in the neighborhood of the transition curves because $\sigma^{2}+\Lambda_{p q}$ is the same order as $2 \epsilon \sigma \gamma_{1}-4 \epsilon \sigma \gamma_{2}$. This is the reason Eqs. (22), (23), (48), and (49) were combined into Eqs. (55) and (56), which were used to determine an expansion valid on and near the transition curves.

\section{The case $\omega$ near $\omega_{q}-\omega_{p}$}

In this case $\omega$ is near $\omega_{q}-\omega_{p}$, and there are no other resonances. The results can be obtained from those above by simply changing the sign of $\omega_{p}$.

\section{The case $\omega$ near $\omega_{p}+\omega_{1 q}$ and $2 \omega$ near $\omega_{s}-\omega_{q}$}

It is convenient to define two detuning parameters $\sigma_{1}$ and $\sigma_{2} ; \sigma_{1}$ is defined according to Eq. (19), while $\sigma_{2}$ is defined according to the following equation:

$$
2 \omega=\omega_{s}-\omega_{q}+\epsilon^{2} \sigma_{2} \text {. }
$$

Then the troublesome terms are eliminated if

$$
\begin{aligned}
& 2 i \omega_{p} D_{2} A_{p}+D_{1}^{2} A_{p}+2 \omega_{p} \hat{\chi}_{p} A_{p}=0, \\
& 2 i \omega_{q} D_{2} A_{q}+D_{1}^{2} A_{a}+2 \omega_{q} \hat{\chi}_{q} A_{q}+2 \omega_{q} \mu_{e s} A_{s} \exp \left(-i \sigma_{2} T_{2}\right)=0,
\end{aligned}
$$

$$
i D_{2} A_{s}+\mu_{s q} A_{q} \exp \left(i \sigma_{2} T_{2}\right)=0
$$

and for $n \neq p, q$, or $s$ the $A_{n}$ satisfy Eq. (37).

Substituting Eqs. (51) and (52) into Eqs. (67) and (68) yields

$$
\begin{aligned}
& 2 i \omega_{p} D_{2} A_{p}+\left(\frac{1}{4} \Lambda_{p q}+2 \omega_{p} \hat{\chi}_{p}\right) A_{p} \\
& -\left(\sigma_{1} f_{p q} / 2 \omega_{p}\right) \bar{A}_{q} \exp \left(i \sigma_{1} T_{1}\right)=0
\end{aligned}
$$

and

$$
\begin{aligned}
& 2 i \omega_{q} D_{2} A_{q}+\left(\frac{1}{4} \Lambda_{p Q}+2 \omega_{q} \hat{\chi}_{q}\right) A_{q}-\sigma_{1} f_{q p} \bar{A}_{p} \exp \left(i \sigma_{1} T_{1}\right) \\
& +2 \omega_{q} \mu_{q s} A_{s} \exp \left(i \sigma_{2} T_{2}\right)=0 .
\end{aligned}
$$

It can readily be shown that Eqs. (22) and (70) result from a multiple-scales expansion of

$$
\begin{aligned}
& 2 i \omega_{p} \frac{d A_{p}}{d t}+\epsilon f_{p q}\left(1-\frac{\epsilon \sigma_{1}}{2 \omega_{p}}\right) \bar{A}_{q} \exp \left(i \epsilon \sigma_{1} t\right)+\epsilon^{2}\left(\frac{1}{4} \Lambda_{p q}\right. \\
& \left.+2 \omega_{p} \hat{\chi}_{p}\right) A_{p}=0 .
\end{aligned}
$$

Equations (23) and (71) result from

$$
\begin{aligned}
& 2 i \omega_{a} \frac{d A_{q}}{d t}+\epsilon f_{q p}\left(1-\frac{\epsilon \sigma_{1}}{2 \omega_{q}}\right) \bar{A}_{p} \exp \left(i \epsilon \sigma_{1} t\right)+\epsilon^{2}\left(\frac{1}{4} \Lambda_{p q}+2 \omega_{q} \hat{\chi}_{q}\right) A_{q} \\
&+\epsilon^{2} \omega_{q} \mu_{q s} A_{s} \exp \left(-i \epsilon^{2} \sigma_{2} t\right)=0,
\end{aligned}
$$

and Eqs. (17) and (69) result from

$$
i \frac{d A_{s}}{d t}+\epsilon^{2} \mu_{s q} A_{q} \exp \left(i \epsilon^{2} \sigma_{2} t\right)=0 .
$$

Equations (72)-(74) admit a nontrivial solution having the form

$$
\begin{aligned}
& A_{p}=a_{p} \exp \left[i \epsilon\left(\lambda+\sigma_{1}\right) t\right], \\
& A_{q}=a_{q} \exp (-i \epsilon \bar{\lambda} t),
\end{aligned}
$$

and

$$
A_{s}=a_{s} \exp \left[-i \epsilon\left(\bar{\lambda}-\epsilon \sigma_{2}\right) t\right],
$$

where $a_{p}, a_{q}$, and $a_{s}$ are complex constants and

$$
\begin{aligned}
\lambda^{3}+ & {\left[\sigma_{1}+\epsilon\left(\gamma_{1}-\sigma_{2}\right)\right] \lambda^{2}+\left[\frac{1}{4} \Lambda_{p q}+\epsilon \sigma_{1}\left(\gamma_{2}-\sigma_{2}\right)\right] \lambda } \\
& -\frac{1}{4} \epsilon \sigma_{2} \Lambda_{p q}=0 .
\end{aligned}
$$

The transition curves correspond to the values of $\epsilon$ and $\omega$ for which $\lambda$ has two equal roots.

As $\epsilon$ vanishes, both resonances cannot exist simultaneously. When $\omega$ approaches $\omega_{p}+\omega_{a}, \sigma_{2}$ becomes very large and Eq. (76) reduces to

$$
\lambda^{2}+\sigma_{1} \lambda+\frac{1}{4} \Lambda_{p q}=0
$$

and, consequently,

$$
\lambda=-\frac{1}{2}\left[\sigma_{1} \pm\left(\sigma_{1}^{2}-\Lambda_{p q}\right)^{1 / 2}\right],
$$

which agrees with Eq. (25). When $2 \omega$ approaches $\omega_{s}$ $-\omega_{q}, \sigma_{1}$ becomes very large and Eq. (76) reduces to $\lambda$ $=0$, indicating that all solutions are bounded. This result agrees with Eq. (47), which shows that for the case of $2 \omega$ near $\omega_{s}-\omega_{a}$ and no other resonances, as $\epsilon$ vanishes, the unstable region in the $\epsilon-\omega$ plane vanishes more rapidly than for the case of $\omega$ near $\omega_{p}+\omega_{q}$.

The case for $\omega$ near $\omega_{p}+\omega_{q}$ and $2 \omega$ near $\omega_{q}+\omega_{s}$ cannot be obtained from the results in this section by changing the sign of $\omega_{q}$ because there is an extra resonance ( $\omega$ near $\left.\omega_{s}-\omega_{p}\right)$ at first order. This case is consider ed below. 


\section{E. The case $\omega$ near $\omega_{p}+\omega_{q}$ and $\omega_{s}-\omega_{q}$}

In this case, $\omega$ is simultaneously near $\omega_{p}+\omega_{q}$ and $\omega_{s}$ $-\omega_{a}$ and thus $2 \omega$ is near $\omega_{p}+\omega_{s}$; there are no other resonances to this order. The troublesome terms are eliminated from Eq. (36) if

$$
2 i \omega_{p} D_{2} A_{p}+D_{1}^{2} A_{p}+2 \omega_{p} \hat{\chi}_{p} A_{p}+2 \omega_{p} \mu_{p s} \bar{A}_{s} \exp \left[i\left(\sigma_{1}+\sigma_{2}\right) T_{1}\right]=0
$$

$2 i \omega_{q} D_{2} A_{q}+D_{1}^{2} A_{q}+2 \omega_{q} \hat{\chi}_{q} A_{q}=0$,

$2 i \omega_{s} D_{2} A_{s}+D_{1}^{2} A_{s}+2 \omega_{s} \hat{\chi}_{s} A_{s}+2 \omega_{s} \mu_{s p} \bar{A}_{p} \exp \left[i\left(\sigma_{1}+\sigma_{2}\right) T_{1}\right]=0$.

Using Eqs. (31)-(33), one finds that

$$
\begin{aligned}
D_{1}^{2} A_{p}= & -\frac{\sigma_{1} f_{p q}}{2 \omega_{p}} \bar{A}_{q} \exp \left(i \sigma_{1} T_{1}\right)+\frac{1}{4} \Lambda_{p q} A_{p} \\
& +\frac{f_{p q} f_{a s}}{4 \omega_{p} \omega_{q}} \bar{A}_{s} \exp \left[i\left(\sigma_{1}+\sigma_{2}\right) T_{1}\right] \\
D_{1}^{2} A_{q}= & \frac{1}{4}\left(\Lambda_{p q}-\Lambda_{q s}\right) A_{q}-\frac{\sigma_{1} f_{a p}}{2 \omega_{q}} \bar{A}_{p} \exp \left(i \sigma_{1} T_{1}\right) \\
& +\frac{\sigma_{2} f_{a s}}{2 \omega_{q}} A_{s} \exp \left(-i \sigma_{2} T_{1}\right) \\
D_{1}^{2} A_{s}= & -\frac{\sigma_{2} f_{a q}}{2 \omega_{s}} A_{q} \exp \left(i \sigma_{2} T_{1}\right)-\frac{1}{4} \Lambda_{q s} A_{s} \\
& -\frac{f_{s q} f_{a p}}{4 \omega_{q} \omega_{s}} \bar{A}_{p} \exp \left[i\left(\sigma_{1}+\sigma_{2}\right) T_{1}\right] .
\end{aligned}
$$

Using Eqs. (80)-(82) in Eqs. (77)-(79), one finds that the resulting equations together with Eqs. (31)-(33) are the first two terms in a multiple-scales expansion of

$$
\begin{aligned}
2 i \omega_{p} \frac{d A_{p}}{d t} & +\epsilon f_{p a}\left(1-\frac{\epsilon \sigma_{1}}{2 \omega_{p}}\right) \bar{A}_{q} \exp \left(i \epsilon \sigma_{1} t\right)+\epsilon^{2}\left(\frac{1}{4} \Lambda_{p q}+2 \omega_{p} \hat{\chi}_{p}\right) A_{p} \\
& +\epsilon^{2}\left(2 \omega_{p} \mu_{p s}+\frac{f_{a p} f_{a s}}{4 \omega_{p} \omega_{q}}\right) \bar{A}_{s} \exp \left[i \epsilon\left(\sigma_{1}+\sigma_{2}\right) t\right]=0
\end{aligned}
$$

$$
\begin{aligned}
2 i \omega_{q} \frac{d A_{q}}{d t} & +\epsilon f_{a p}\left(1-\frac{\epsilon \sigma_{1}}{2 \omega_{q}}\right) A_{p} \exp \left(i \epsilon \sigma_{1} t\right)+\epsilon f_{q s}\left(1+\frac{\epsilon \sigma_{2}}{2 \omega_{q}}\right) A_{s} \\
& \times \exp \left(-i \epsilon \sigma_{2} t\right)+\epsilon^{2}\left(\frac{1}{4} \Lambda_{p q}-\frac{1}{4} \Lambda_{q s}+2 \omega_{q} \hat{\chi}_{q}\right) A_{q}=0,
\end{aligned}
$$

$$
\begin{aligned}
2 i \omega_{s} \frac{d A_{s}}{d t} & +\epsilon f_{s q}\left(1-\frac{\epsilon \sigma_{2}}{2 \omega_{s}}\right) A_{a} \exp \left(i \epsilon \sigma_{2} t\right) \\
& +\epsilon^{2}\left(2 \omega_{s} \mu_{s p}-\frac{f_{s q} f_{a p}}{4 \omega_{q} \omega_{s}}\right) \bar{A}_{p} \exp \left[i \epsilon\left(\sigma_{1}+\sigma_{2}\right) t\right] \\
& +\epsilon^{2}\left(2 \omega_{s} \hat{\chi}_{s}-\frac{1}{4} \Lambda_{q s}\right) A_{s}=0 .
\end{aligned}
$$

Equations (83)-(85) admit nontrivial solutions having the form

$$
\begin{aligned}
& A_{p}=a_{p} \exp \left[i \epsilon\left(\lambda+\sigma_{1}\right) t\right], \\
& A_{q}=a_{q} \exp (-i \epsilon \bar{\lambda} t),
\end{aligned}
$$

and

$$
A_{s}=a_{s} \exp \left[-i \epsilon\left(\bar{\lambda}-\sigma_{2}\right) t\right]
$$

where $a_{p}, a_{q}$, and $a_{s}$ are complex constants and

$$
\begin{gathered}
\lambda^{3}+\left(\sigma_{1}-\sigma_{2}+\epsilon \gamma_{1}\right) \lambda^{2}+\left[\frac{1}{4}\left(\Lambda_{p q}-\Lambda_{q s}\right)-\sigma_{1} \sigma_{2}+\epsilon\left(\sigma_{1} \gamma_{2}+\sigma_{2} \gamma_{3}\right)\right] \lambda \\
-\frac{1}{4}\left(\sigma_{1} \Lambda_{q s}+\sigma_{2} \Lambda_{p q}\right)+\epsilon\left(\sigma_{1} \sigma_{2} \gamma_{4}+\gamma_{5}\right)=0
\end{gathered}
$$

where

$$
\begin{aligned}
& \gamma_{1}=\frac{1}{8}\left[\Lambda_{p q}\left(\frac{1}{\omega_{q}}-\frac{1}{\omega_{p}}\right)-\Lambda_{q s}\left(\frac{1}{\omega_{s}}+\frac{1}{\omega_{q}}\right)-\hat{\chi}_{p}+\hat{\chi}_{q}+\hat{\chi}_{s}\right], \\
& \gamma_{2}=-\frac{1}{8}\left(\frac{\Lambda_{p q}}{\omega_{p}}+\frac{\Lambda_{a s}}{\omega_{q}}+\frac{\Lambda_{a s}}{\omega_{s}}\right)+\hat{\chi}_{q}+\hat{\chi}_{s}, \\
& \gamma_{3}=\frac{1}{8}\left(\frac{\Lambda_{s q}}{\omega_{s}}+\frac{\Lambda_{p q}}{\omega_{p}}-\frac{\Lambda_{p q}}{\omega_{q}}\right)+\hat{\chi}_{p}-\hat{\chi}_{a s} \\
& \gamma_{4}=\frac{1}{8}\left(\frac{\Lambda_{p q}}{\omega_{p}}+\frac{\Lambda_{a s}}{\omega_{s}}\right)-\hat{\chi}_{a s}
\end{aligned}
$$

and

$$
\begin{aligned}
\gamma_{5}= & \frac{1}{32} \Lambda_{q s} \Lambda_{p Q}\left(\frac{1}{\omega_{s}}-\frac{1}{\omega_{p}}\right)+\frac{1}{4}\left(\Lambda_{q s} \hat{\chi}_{p}+\Lambda_{p q} \hat{\chi}_{s}\right) \\
& -\frac{1}{4 \omega_{q}}\left(\frac{\mu_{s p} f_{p Q} f_{q s}}{\omega_{p}}+\frac{\mu_{p s} f_{q p} f_{s Q}}{4 \omega_{s}}\right) .
\end{aligned}
$$

The transition curves correspond to the values of $\epsilon$ and $\omega$ for which there are two equal roots.

When $\epsilon$ vanishes, Eq. (87) reduces to the results for a single resonance.

\section{EXAMPLE}

Here we consider the response of a free-clamped column to a sinusoidally varying axial force. This is one of the examples considered by Iwatsubo et al. , ${ }^{8}$ who obtained two-term expansions of the transition curves and did not consider the simultaneous occurrence of two resonances. We compare their results with the present results, showing the influence of the third term in the expansion and the effect of the occurrence of simultaneous resonances.

Iwatsubo et al. showed how the equations governing the motion of the column can be put in the form of Eq. (1). We note that the coefficients of the forcing term are defined differently;

$$
f_{i j}=\frac{1}{2} b_{i j},
$$

where the $b_{i j}$ are given by Iwatsubo et al. Moreover, Eq. (10) given in their paper has typographical errors; the first three terms should have the subscript $k$ and the last term should read

$$
\beta \gamma^{2} \sum_{j=1}^{\infty} b_{j k} f_{j} \cos \theta t .
$$

Figure 1 shows the transition curves. The results for $\omega$ near $2 \omega_{1}$ and $\omega_{2}-\omega_{1}$ were computed by using Eq. (63), which applies when there is a single resonance. The results for $\omega$ near $\omega_{3}-\omega_{2}, 2 \omega_{2}, \omega_{4}-\omega_{3}$, and $\omega_{1}+\omega_{3}$ were computed by using both Eq. (63) (these are shown by dotted lines) and Eq. (87) (these are shown by solid lines); the latter applies when multiresonances occur simultaneously. For the present results, twenty terms were used to compute $\mu_{p q}, \mu_{q s}, \chi_{p}, \chi_{q}$, and $\chi_{s}$. For the case when $\omega$ is near $2 \omega_{2}$ and $\omega_{3}-\omega_{2}$ simultaneously, the 


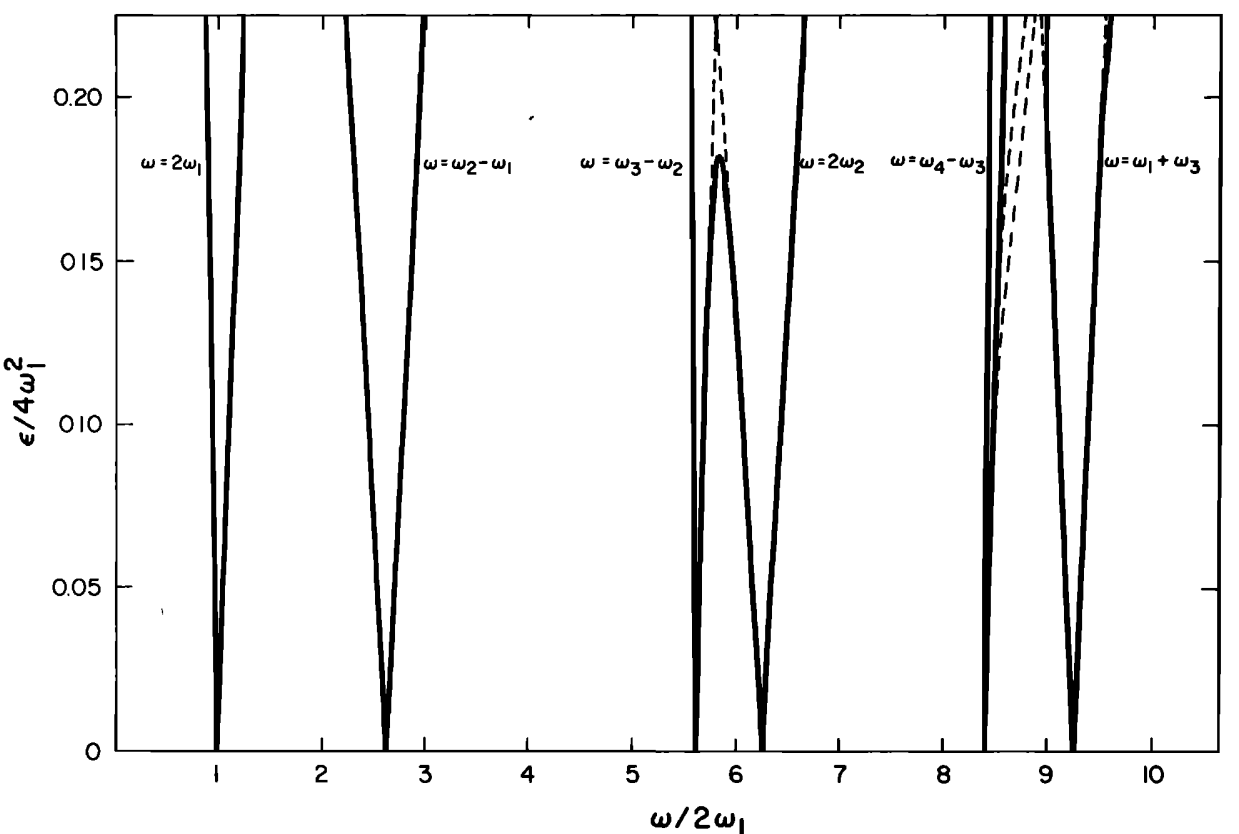

FIG. 1. Transition curves for the free-clamped column. results obtained from Eq. (87) show a rounded merger lower than the intersection obtained from Eq. (63). For the case when $\omega$ is near $\omega_{4}-\omega_{3}$ and $\omega_{1}+\omega_{3}$ simultaneously, the results obtained from Eq. (87) do not intersect in contrast with the results obtained from Eq. (63).

We note that there is no discernible difference between the two sets of present results [Eqs. (63) and (87)] and between the present results and those of Iwatsubo $e t a l$. when $\epsilon$ is less than 0.1. Generally, the effect of the third term is to bend the transition curves slightly to the right.

\section{CONCLUDING REMARKS}

The method of multiple scales is used to determine uniformly valid asymptotic solutions for parametric excitations of systems having multiple degrees of freedom. The results show that resonances occur when $p \omega \approx \omega_{n}$ $\pm \omega_{m}$, where $p, n$, and $m$ are integers. Explicit expressions for the characteristic exponents on and near the transition curves are obtained for several possible resonant conditions. In some cases, the present results are found to agree with the results obtained earlier by others, while other cases are considered here for the first time. Though the example considered in the present paper is somewhat limited in its generality, the method is not and can be readily applied to other similar systems, including those with weak nonlinearities and general periodic excitations.

Finally, it is noted that in the case of disjoint resonances, such as $\omega \approx \omega_{n} \pm \omega_{m}$ and $\omega \approx \omega_{s} \pm \omega_{r}$ with $m$ and $n$ being different from $r$ and $s, u_{r}$ and $u_{s}$ may be unbounded along transition curves for $u_{n}$ and $u_{m}$ and vice versa. Hence, the transition curves in the case of disjoint res- onances are the pair obtained from one of these resonances along which all other solutions are bounded.

${ }^{1}$ C. S. Hsu, "On the parametric excitation of a dynamic system having multiple degrees of freedom," J. Appl. Mech. 30, 368 (1963).

${ }^{2}$ C. S. Hsu, "Further results on parametric excitation of a dynamic system," J. Appl. Mech. 32, 373 (1965).

${ }^{3}$ T. Yamamoto and A. Saito, "On the vibration of "Summed and differential types' under parametric excitation," Nagoya University Faculty of Engineering Memoirs 22, 54 (1970).

${ }^{4} \mathrm{E}$. Mettler, Dynamic Stability of Structures, edited by G. Herrmann (Pergamon, New York, 1967), p. 169.

${ }^{5}$ A. H. Nayfeh, Perturbation Methods (Wiley, New York, 1973).

${ }^{6} \mathrm{Y}$. Sugiyama, N. Fujiwara, and T. Sekiya, "Studies on nonconservative problems of instability of columns by means of of an analogue computer," Proceedings of the 18th Japan $\mathrm{Na}-$ tional Congress for Applied Mechanics (Central Scientific, Toyoko, 1970) p. 113.

${ }^{7}$ T. Iwatsubo, M. Saigo, and Y. Sugiyama, "Parametric instability of clamped-clamped and clamped-simply supported columns under periodic axial loads," J. Sound Vib. 30, 65 (1973).

${ }^{8} \mathrm{~T}$. Iwatsubo, Y. Sugiyama, and S. Ogino, "Simple and combination resonances of columns under periodic axial loads," $\mathrm{J}$. Sound Vib. 33, 211 (1974).

${ }^{9}$ F. C. Fu and S. Nemat-Nasser, "On stability of solution of systems of linear differential equations with harmonic coefficients," ALAA J. 10, 30 (1972).

${ }^{10} \mathrm{~K}$. G. Valeev, "On Hill's method in the theory of linear differential equations with periodic coefficients," Prikl. Mat. Mekh. 24, 979 (English transl.: 1493).

${ }^{11} \mathrm{~K}$. G. Valeev, "On Hill's method in the theory of linear differential equations with periodic coefficients. Determination of the characteristic exponents," Prikl. Mat. Mekh. 25, 314 (English transl.: 1460).

${ }^{12} \mathrm{~K}$. G. Valeev, "On the danger of combination resonance," Prikl. Mat. Mekh. 27, 1134 (English transl.: 1745). 